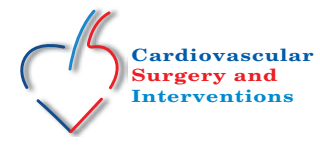

\title{
A left atrial thrombus forming an antibioma: a rare entity
}

\author{
Trushar Gajjar, Nageswar Rao, Neelam Desai \\ Sri Sathya Sai Institute of Higher Medical Sciences - Prasanthigram, CTVS Department, Prasanthigram, Andhra Pradesh, India
}

Received: July 13, 2016 Accepted: July 28, 2016 Published online: January 11, 2017

\section{ABSTRACT}

An antibioma is defined as a hard edematous swelling containing sterile pus following treatment of an abscess with long-term antibiotherapy. Abscess of the heart, as any abscess elsewhere in the body, develops in two principal ways: (i) by dissemination from a distant infectious focus (ii) by direct extension of an infectious process located in the heart itself. Even the fresh blood clot in the heart may become infected. Herein, we report the first case of the left atrial thrombus forming an antibioma following the Lutembacher's repair.

Keywords: Antibioma; Lutembacher's syndrome; mitral valve replacement; thrombus left atrial appendage.

Antibioma, which is a rare anomaly of the heart, is defined as a hard edematous swelling containing sterile pus following treatment of an abscess with long-term antibiotherapy. In the literature, a left atrial (LA) antibioma has never been reported. Herein, we present an unusual case of the LA thrombus forming an antibioma following the Lutembacher's repair.

\section{CASE REPORT}

A 28-year-old female was admitted with complaints of dyspnea on exertion. She was in the New York Heart Association Class II (NYHA II) for four years. Through a detailed examination, she was diagnosed with Lutembacher's syndrome. A two-dimensional echocardiography revealed ostium secundum atrial septal defect and severe mitral stenosis. There was no history of pulmonary tuberculosis or thrombus in the left atrium or appendage. Ostium secundum atrial septal defect was closed with an autologous-untreated pericardial patch and mitral valve was replaced with a 29-tilting disc mechanical prosthetic heart valve. Surgery was uneventful. On postoperative Day 2, the patient developed high-grade fever $\left(38^{\circ} \mathrm{C}\right)$. Blood culture showed a heavy growth of Streptococcus which was treated according to Streptococcus-sensitive antibiotics. She was extubated in the fifth postoperative day. The patient was on an anticoagulant with international normalized ratio (INR) set at 2.5 to 3.0. In the ninth postoperative day, transthoracic echocardiography showed a large LA thrombus (Figure 1), despite oral anticoagulants and target INR. She was, then, put on heparin; however, repeated echocardiography did not show any improvement. The patient developed tachypnea and chest X-ray and computed tomography (CT) scan showed patchy confluent areas of consolidation in both the lungs, more dense in the lower lobes. Thoracic CT also showed mosaic perfusion along with the areas of ground-glass changes in both the lungs with an organized thrombus in the posterior part of the LA (Figure 2) measuring $4 \times 3 \mathrm{~cm}$ in size without a thrombus in the LA appendage. The patient was scheduled for LA clot removal. Intraoperatively, there was a large, $4 \times 3 \times 3 \mathrm{~cm}$, well-defined globular mass with very thin glistening covering of the left atrium just beneath the right inferior pulmonary vein away from the prosthetic valve and the patch (Figure 3). During manipulation, the mass ruptured and turbid brown-colored fluid came out. The wall of the mass was excised and pus was drained. The pus was sent for culture, which did not grow any organism initially. The LA was cleaned with normal saline and vancomycin. Following the redo surgery, the patient required tracheostomy due to prolonged intubation and she continued to have spikes of fever with blood culture growing Candida albicans. On Day 118 days of the intensive care unit (ICU) stay, the patient developed multi-organ failure secondary to

Corresponding author: Trushar Gajjar, MD. Sri Sathya Sai Institute of Higher Medical Sciences - Prasanthigram, CTVS Department, Prasanthigram, 515134 Andhra Pradesh, India.

Tel: +91 9700355411 e-mail: trushargajjar@gmail.com 

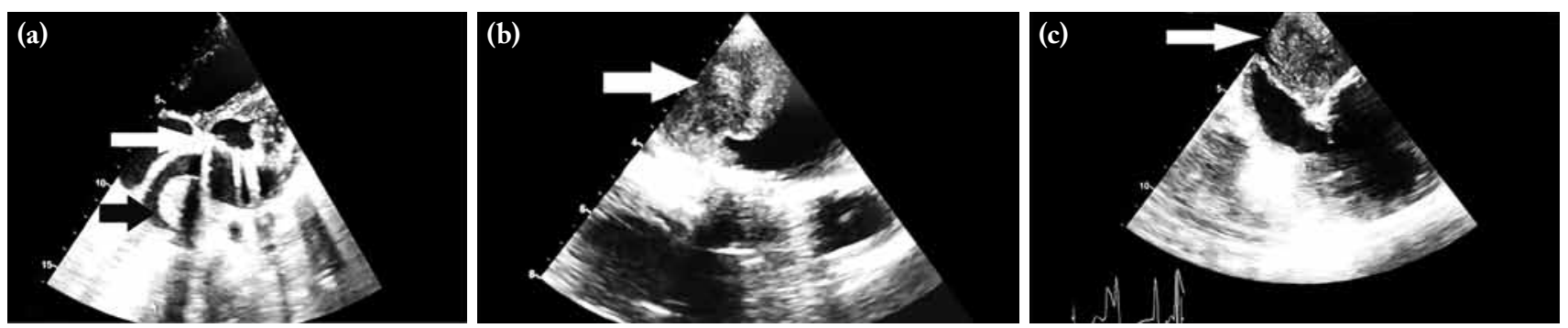

Figure 1. Echocardiography. (a) Transthoracic echocardiography showing the prosthetic valve (white arrow) and the left atrial thrombus (black arrow). (b) Transesophageal echocardiography showing the left atrial thrombus (white arrow). (c) Transesophageal echocardiography showing the left atrial thrombus (white arrow).

fungal septicemia. Histopathologica report suggested an infected thrombus and fluid culture was reported as the growth of Stenotrophomonas maltophilia.

\section{DISCUSSION}

Antibioma of the heart is a rare occurrence which has not yet been documented in the literature. It is defined as a hard edematous swelling containing sterile pus following the treatment of an abscess with longterm antibiotherapy. ${ }^{[3]}$ Abscess of the heart, as any abscess elsewhere in the body, develops due to (i) the dissemination from a distant infectious focus, (ii) the direct extension of an infectious process located in the heart itself, ${ }^{[1]}$ or (iii) fresh blood clot in the heart becoming infected, as in our case. ${ }^{[1-3]}$ Prosthetic valve
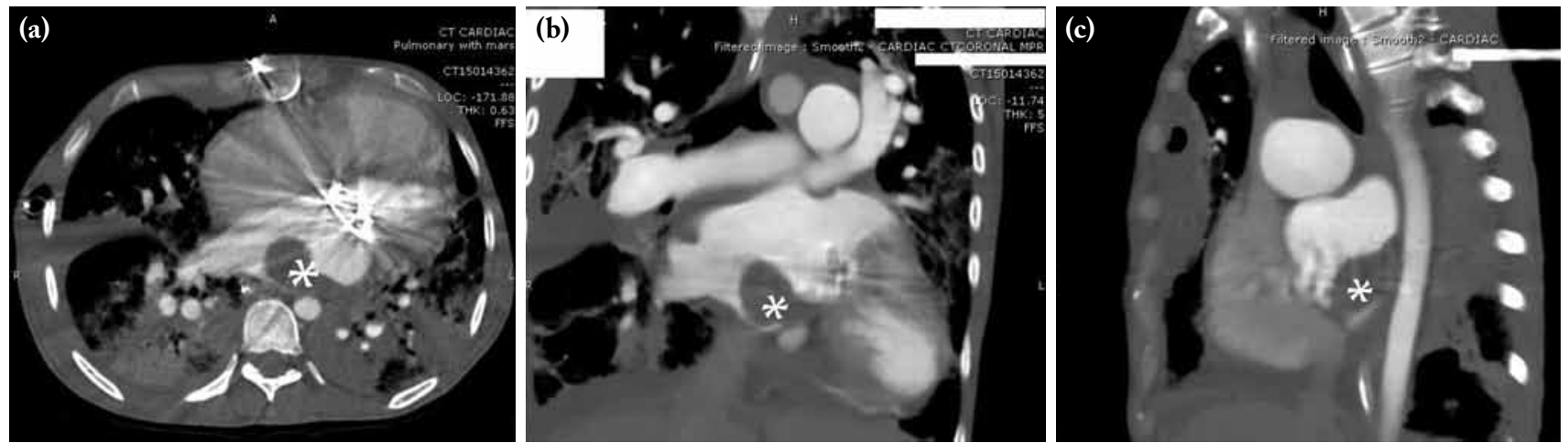

Figure 2. Computed tomography scans. (a) A coronal section showing prosthetic valve and the left atrial thrombus $(*)$. (b) A sagittal section showing the left atrial thrombus $(*)$. (c) A lateral view showing the left atrial thrombus $\left(^{*}\right)$.
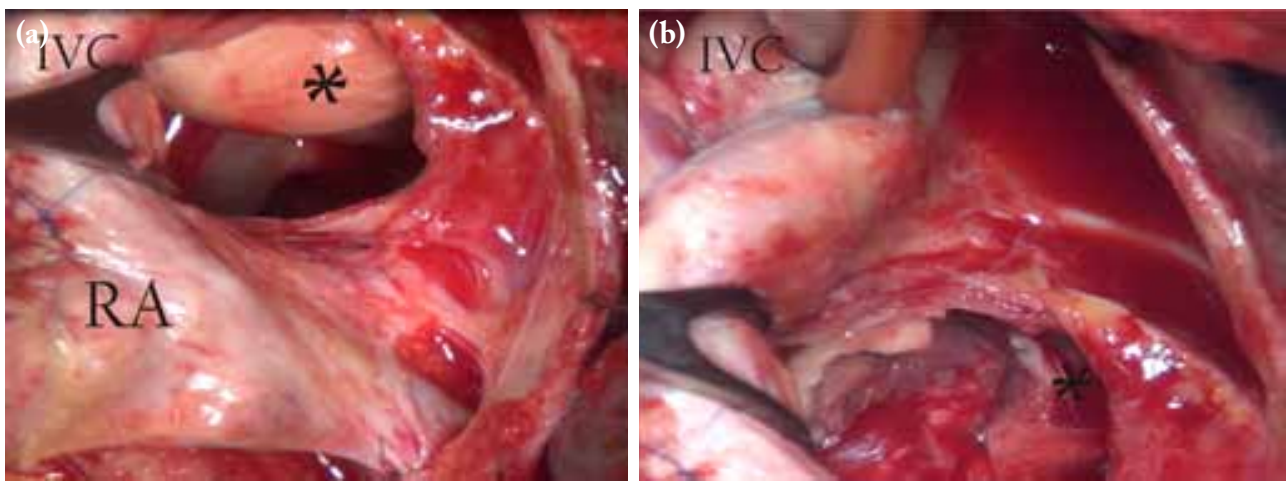

Figure 3. Intraoperative images. (a) An intraoperative image showing an opened left atrium and antibioma $\left(^{*}\right)$. (b) An intraoperative image showing the debrided area of the left atrial antibioma $\left(^{*}\right)$. IVC: Inferior vena cava; RA: Right atrium. 
endocarditis can lead to abscess formation; however, it is usually limited to the annulus and may infect the newly formed clot in the vicinity. ${ }^{[2]}$ In our case, we initially suspected of prosthetic valve endocarditis; however, prosthetic valve appeared normal and a new thrombus formed at the posterior wall of left atrium, near the right inferior pulmonary vein. The main cause probably would be (i) damage to the endothelium and raw exposed muscle with persistent atrial fibrillation would have become the source for the $\operatorname{clot}^{[3]}$ or (ii) the atrial septal defect closure with a pericardial patch would have left suture or raw pericardial tissue at the lower most end of the patch, which might have formed the clot which became infected over a period of time due to prolonged ICU stay and hospital-acquired infection, or (iii) possibility of very low cannulation of inferior vena cava and deep reinforcement of the suture would form the pocket where the blood became stagnated and formed a thrombus at the lower end of the interatrial septum, in particular. This was probably the cause in our case. In such cases, diagnosis can be obtained by echocardiography and antibioma can be suspected, if the clot is in unusual position, as in our case, and does not respond to intravenous heparin. Computed tomography offers the exact location and the content of the mass. Surgical evacuation thorough normal saline wash with antibiotics is the primary treatment of choice. However, the postoperative course remains stormy and outcome may be dismal.

In conclusion, antibioma of the left atrium is a very rare occurrence with no published literature to date. Therefore, we believe that early recognition and aggressive management may yield satisfactory outcomes.

\section{Declaration of conflicting interests}

The authors declared no conflicts of interest with respect to the authorship and/or publication of this article.

\section{Funding}

The authors received no financial support for the research and/or authorship of this article.

\section{REFERENCES}

1. Zettner A, Irmiere VJ. Abscess of the heart: a complication of acute vegetative and ulcerative endocarditis. Circulation 1959;20:590-4.

2. Cabell CH, Abrutyn E, Karchmer AW. Cardiology patient page. Bacterial endocarditis: the disease, treatment, and prevention. Circulation 2003;107:e185-7.

3. Raut MS, Maheshwari A, Shad S. Real and pseudo clot in left atrial appendage. Ann Card Anaesth 2014;17:42-3. 